\title{
A Comparative Analysis of Shape-Based and Zernike Moment Feature Extraction Techniques for Fasteners Recognition Using Neural Network
}

Nur Diyanah Mustaffa Kamal

Nor'aini Jalil

Hadzli Hashim

\begin{abstract}
This paper presents a Comparative Analysis of Shape-based and Zernike moment Feature Extraction Techniques for Fastener Recognition. There a nine features extracted using shape-based technique and 64 moments used in Zernike feature extraction technique. For Zernike moment technique, the 64 moments are divided into 3 groups. The first group is the lower order moments, the second group is the higher order moments and the third group is the combination between the lower order group and the higher order group. The processes taken in the recognition are image acquisition, pre-processing, segmentation, feature extraction, and classification. The segmentation process is carried out by using adaptive filter and the classification process employed artificial neural network. The final result from this experiment is that shape-based technique has a better classification result of about $84.93 \%$ correct recognition compared to Zernike moment technique which is about $\mathbf{5 1 . 5 3 \%}$ (combined group).
\end{abstract}

Keywords-Shape-based technique, Zernike moment, fastener recognition, artificial neural network.

\section{Introduction}

Fasteners comes in many forms, shapes and sizes. Examples of types of fasteners existed today are screw, nail, stud, nut and bolt. From these types of fasteners, there are thousands that are uniquely different standard from each other. For screw, the difference are due to major, minor and pitch diameter of the threading around the screw [1]. Using specific fastener to assemble object is very important especially in building vehicle like cars and planes. It is extremely dangerous if fasteners are used without care on these type of objects. The fasteners may be loosely fit and cause accidental disassembly which can occur during on the road or on the flight. To avoid such incident happens, each specific fasteners must be used. Here, fastener recognition is needed because in a manufacturing factory, millions of fasteners are used. Often in workshops, different fasteners are mixed up with each other. This is when fastener recognition is needed which is to recognize the fastener individually using computer vision.

The earliest system for recognition of fastener is a screw inspection device [2]. This invention was to solve the problem of accidently feeding defective fastener in an automatic screw fastener. The system has sensors to measure head diameter, shank, and thread length. Another similar system was invented which was the automated fastener inspection system [3]. Fasteners which are moving through a feeding line are inspected by using a camera which produce video image of stopped fastener. The extracted features from the images are grip and thread length, fastener diameter and angularity of fastener's head. Fastener verification system was then invented as described in [4] to help in aviation industry where high volume fastening machines were used. The problem arise from rapid fastening machine are caused by defective rivets that were fed in and sometimes caused serious problem which led to replacement of the whole aircraft wing. This invention has helped in term of verification of correct fastener to be used. The features that is extracted from the fastener are length and diameter. These systems have similar drawbacks such as neglected colour feature and can only recognise one specific screw. In [5], Artificial Neural Network (ANN) is used to recognise bolt and nut. The recognition is done by using image processing in real time. $92 \%$ success recognition rate was achieved using this method. The feature that is extracted from this system is minimum and maximum of radius distance's curve of the fastener. Later, the Industrial Strength Pipeline invented a system for Recognizing Fastener [6]. The system recognises fasteners based on the length, width, base diameter, and thread counts. Classification is done by using support vector machine (SVM). However, the success rate is only $50 \%$ due to small different between grade types.

Through the reviews of the feature extraction techniques, the shaped- based technique is not commonly being used especially for fasteners recognition and Zernike moment is frequently being used for face recognition. For these reasons these two techniques are used to investigate its effectiveness in identifying the fasteners.

This paper is organized as follows. Previous works in fasteners' feature extraction techniques is discussed in the Introduction. Explanation about shape-based and Zernike moment feature extraction techniques is discussed in section 2. Section 3 discussed about the methodology used in image acquisition, pre-processing, processing, feature extraction and classification. Section 4 discussed about the experimental result of the classification performance when using these two techniques and lastly section 5 concludes the overall findings.

\section{Shape-based and Zernike Moment Feature Extraction Techniques}

\section{A. Shape-Based Feature Extraction Technique}

The objective of feature extraction is to identify different attributes that makes the fastener unique from the others. Shape-based technique is by measuring the fastener in term of pixel values. The features are eccentricity, equivalent diameter, convex area, area, extent, major axis length, minor axis length, perimeter and solidity of the fastener. These nine features are measured by using MATLAB R2013a. The list below shows the meaning of each features used. These 
features are used along the 'regionprops' command which measure attributes of image region [7].

- Eccentricity: Ratio of the length between the foci of the ellipse and the region's major axis length [8].

$$
\text { Eccentricity }=\sqrt{\frac{\lambda_{\max }}{\lambda_{\min }}}
$$

Where $\lambda_{\max }$ and $\lambda_{\min }$ are the eigenvalues of the matrix

$$
\begin{aligned}
& {\left[\begin{array}{ll}
\mu_{2,0} & \mu_{1,1} \\
\mu_{1,1} & \mu_{0,2}
\end{array}\right]} \\
& \mu_{p, q}=\sum x \sum y((x-\bar{x}))^{p}(y-\bar{y})^{q}
\end{aligned}
$$

Where $x$ and $y$ are coordinate of the region and $\bar{x}$ as well as $\bar{y}$ are the centroids.

- Equivalent diameter: The diameter of circle with the same area of the fastener [9].

$$
\text { Equivalent Diameter }=\sqrt{\frac{4 A}{\pi}}
$$

Where $\mathrm{A}$ is the area of the equivalent circle of the fastener.

- Convex area: The number of pixels in convex image [10].

- Area: The total number of pixels of the segmented fastener.

- Extent: Ratio of pixels in the fastener region to the pixels in the total bounding box.

- Major axis length: Length (in pixels) of the major axis of the ellipse that has the same normalized second central moments as the region [7].

- Minor axis length: Length (in pixels) of the minor axis of the ellipse that has the same normalized second central moments as the region [7].

- Perimeter: Number of pixels in the boundary of the foreground region [11].

- Solidity: Solidity expressed how much the shape is concave or convex [12].

$$
\text { Solidity }=\frac{A_{x}}{H}
$$

Where $A_{5}$, is the area of fastener's region and $\mathrm{H}$ is the convex hull area of the foreground.

\section{B. Zernike Moments Feature Extraction Technique}

The second feature extraction technique is Zernike moment. Zernike moment is insensitive to small changes in respect to the original image and importantly this moment is not affected by rotation. Image that is calculated using Zernike moment is mapped to a set of orthogonal basis functions. The image is placed in a unit circle and the coordinate $(0,0)$ is plotted on the centre of the image. Equation below shows the definition of Zernike moment of order $n[13,14$, and 15].

$$
Z_{n m}=\frac{n+1}{\pi} \sum_{\text {over wnit circle }} \sum f(x, y)\left[V_{n m}(\rho, \theta)\right]^{*}
$$

Where $f(x, y)$ is the image's function, $\operatorname{Vnm}(\rho, \theta)$ is the Zernike polynomial which is defined as equation below,

$V_{n m}(x, y)=V_{n m}(\rho, \theta)=R_{n m}(\rho) e^{i m, \theta}$

Where $m$ is the positive and negative integers which follows the rules $\mathrm{n}-|\mathrm{m}|_{\text {even }},|\mathrm{m}|<\mathrm{n} . \rho$ is the distance between origin to $(\mathrm{x}, \mathrm{y})$ pixel. $\theta$ is the angle between vector $\rho$ and $\mathrm{y}$-axis. $\mathrm{R}_{\mathrm{nm}}(\rho)$ is radial polynomial where,

$$
R_{n m}(\rho)=\sum_{s=0}^{\frac{\frac{\pi n-s n}{2}}{2}}(-1)^{s} \frac{(n-s) !}{s !\left(\frac{n+m}{2}-s\right) !\left(\frac{n-m}{2}-s\right)} \rho^{n-2 s}
$$

For the feature extraction method, 32 Zernike moments of lower orders are calculated from an image and is defined as group 1 moment and another 32 Zernike moments of higher order are calculated which is named as group 2 [16]. There are 64 moments to describe an image which consist of 16 orders and 64 repetitions.

\section{Methodology}

The following steps are the process taken in this research;

\section{A. Image Acquisition}

The fasteners used in this research are screws, nails and rivet that are commonly found in local hardware stores. The difference between the samples is in term of size, length and colour. Image acquisition of different fasteners is collected using a digital camera (Canon Eos 1100D) with fixed distant of $11 \mathrm{~cm}$ between the camera's lens and the fastener. The fastener is put onto a white background and the mode of the camera is set to macro. These procedures are done inside a SANOTO lightbox with a shooting area of $296 \times 215 \times 200$ $\mathrm{mm}$. Light box is needed to avoid stray lights entering the image acquisition area. The angle between the camera lens and the fastener is 90 degree. There are 30 different fasteners with each fastener is captured 100 times with random positions and angles. Thus, the total image for this project is 3000 .

\section{B. Image Pre-processing}

The image of fasteners on white background are preprocessed and segmented to enhance the resolution of the image. The pre-processing steps includes decreasing the size of the image proportionally by a factor of 0.3 .The original image dimension is $2256 \times 1504$ pixels and the pre-processed image dimension is $677 \times 452$ pixels. The reduction of image size is to decrease the computation time when the image is processed. After reducing the dimensions of the images, the coloured image is transform from RGB colour space to two dimensions image which is in grayscale. Equation (9) shows the multiplication and addition of $\operatorname{red}(\mathrm{R})$, green $(\mathrm{G})$ and blue (B) component of the image in order to get the grayscale value $(\mathrm{Y})$ of the single pixel.

$Y=0.299 * R+0.587 * G+0.114 * B$ 


\section{Foreground Segmentation}

Adaptive threshold is used to remove the background. This algorithm is used because global threshold could not solve the problem of uneven illumination during the image acquisition process. The figure 1 shows the comparison between global threshold method and adaptive threshold method. Note that the foregrounds in this figure are in black colour.

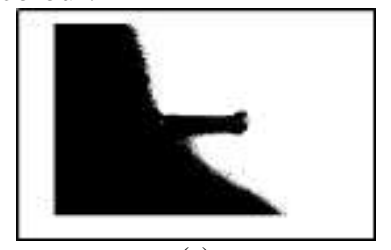

(a)

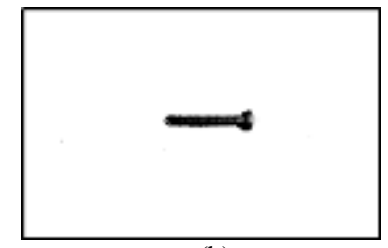

(b)
Figure 1. Comparison between global threshold and adaptive threshold. (a) Global threshold segmentation. (b) Adaptive threshold segmentation.

Global threshold method produce unsatisfactory segmentation of the fastener's image. The shadow of the image that has been captured is recognized as the foreground. The shadow produced is due to the uneven lighting condition in the lightbox. When adaptive threshold is used, the shadow is ignored and only the fasteners' pixels are recognized. Adaptive threshold solve this problem by normalizing the brightness of each pixel to compensate the uneven lighting of the image [17] .There are four parameters that needed to be set for the adaptive threshold algorithm which are the filter size, filter type, threshold mode and percentage $(\mathrm{t})$ relative to the local average grey value below which the threshold value is set. The parameters are $1 / 10$ for filter size, median for filter type, 'relative' for threshold mode and $20 \%$ for parameter ' $t$ '. However, the image produced using these parameters will make the fastener's pixel becomes black and the background becomes white. Thus, the image will be inverted to let the fastener become the foreground (white).Although the fastener has been segmented, imperfections exists such as holes which are the result of highlights on the fastener. To remove this imperfections, morphological operations are done until no hole existed in the structure. Among the morphological process used are closing, opening and filling operations.

\section{Feature Extraction}

After the fastener have been fully segmented, shapebased and Zernike moments feature extraction techniques are applied to get the numerical attributes that describe the fastener. There are 9 attributes for shape-based technique and 64 attributes for Zernike moment technique. The shape based features are eccentricity, equivalent diameter, convex area, area, extent, major axis length, minor axis length, perimeter and solidity of the fastener. For Zernike moment feature, the fastener images are transformed to binary image using the previous method used in extracting shape-based features. Then, the computations of Zernike moment are done on the fasteners' binary images. The absolute values of Zernike moment are taken as the feature descriptor. The computation is repeated from low Zernike moment order to higher order. Comparison between the lower order and higher order are done to seek the best feature for classification stage.

\section{E. Classification}

The classifier used in this research is a single hidden layer feedforward multilayer perceptron artificial neural network. The covariates are normalized and the activation function in the hidden layer is hyperbolic tangent. Equation 10 shows the formula for hyperbolic tangent function with the range of output is from -1 to 1 [18].In the output layer, on the other hand, have the activation function of softmax. Other than that, the error function is in term of crossentropy.

$\tanh (x)=\frac{\sinh (x)}{\cosh (x)}=\frac{e^{x}-e^{-x}}{e^{x}+e^{-x}}$

\section{Experimental Result and Discussion}

There are 30 unique fasteners used in this research. For every fasteners, the number of images taken are 120 times with different positions and angles. Figure 2 bellow shows the sample data taken.
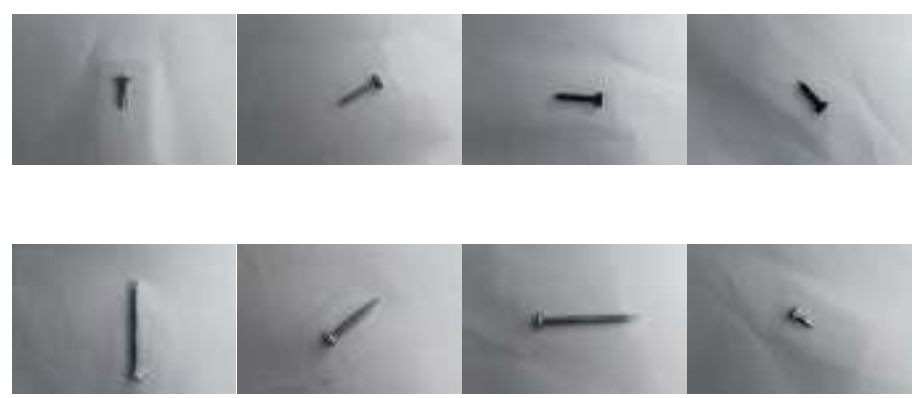

Figure 2. 8 unique fasteners sample images in different angles.

After the images are taken, pre-process steps took place. The images' dimensions are reduced and the colour space is transformed from RGB to grayscale. Then, segmentation process are done until no more unnecessary blobs and background are present. Figure 3 shows the segmented images of the fasteners.
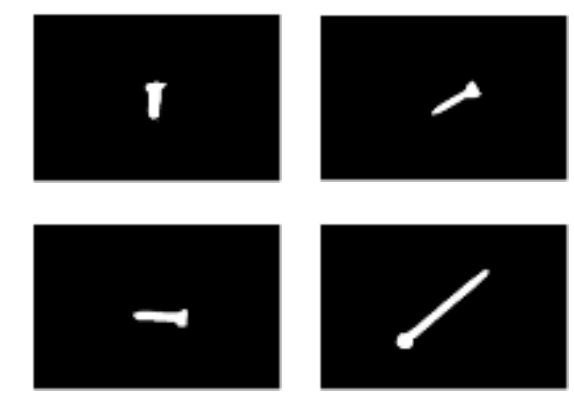

Figure 3. Segmented images of 4 sample fasteners.

Next, the features are extracted by using shape-based technique and Zernike moments. Kruskal-Wallis test was done to test the null hypothesis that the population median of all the groups are equal [19]. All the shape-based features have the value of below 0.05 which mean that all the features are statistically significant. Same goes to Zernike moment features, class 1 , class 2 and combined moments class have Kruskal Wallis value of below than 0.05. If there are features with Kruskal Wallis value more than 0.05 , the 
Proc. of Sixth International Conference On Advances in Computing, Electronics and Electrical Technology - CEET 2016Copyright $@$ Institute of Research Engineers and Doctors. All rights reserved. ISBN no. 978-1-63248-109-2 doi: 10.15224/ 978-1-63248-109-2-17

features need to be removed because insignificant in the classification stage. For shape-based technique classification stage, there are 9 inputs and 30 targets. The data is divided into training and testing set. $40 \%$ ( 1440 images) of the data are fed into the training set and $60 \%$ ( 2160 images) of the data are fed into the testing set. The classification is done 10 times and the average correct classification are calculated. As for Zernike moment, there are three classes which are the group 1, group 2, and combined group. For group 1 and 2, there are 32 inputs and 30 outputs respectively. Combined group have 64 inputs and 30 outputs.

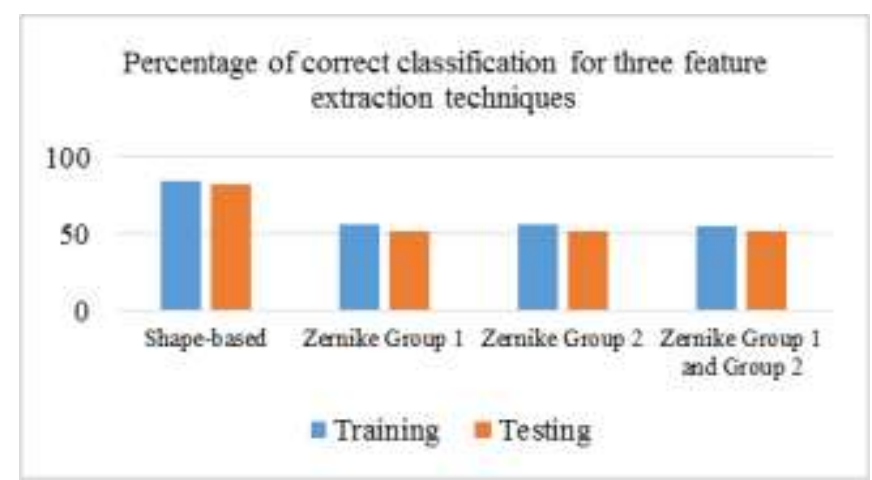

Figure 4. Percentage of correct classification for three feature extraction techniques.

Based on the result, shape-based technique (84.93\% for training and $82.1 \%$ for testing) have significantly higher correct recognition compared to Zernike group $1(56.41 \%$ for training and $51.6 \%$ for testing) and group $2(56.17 \%$ for training and $52.2 \%$ for testing). Even the combined group of 64 Zernike moments (54.86\% for training and $51.53 \%$ for testing) could not give a good result. Interestingly, the percentage of correct prediction dropped when using combination of group 1 and group 2 moments.

\section{v. Conclusion}

In this experiment, shape-based technique outperformed Zernike moment in recognizing the fasteners. Since Zernike moment used is the direct method, therefore it is not efficient enough to recognize fasteners although 64 different moments are used as features and inputs to the neural network while, the shape-based technique utilized only 9 attributes to describe the fasteners. The contribution of this paper are, method used to segment the fastener on white background effectively using adaptive filter and the shapebased technique proof to be more efficient than Zernike moment technique. The percentage of correct predictions for Zernike moments are about $51.6 \%$ for group $1,52.2 \%$ for group 2 and $51.53 \%$ for combined group 1 and 2 . The percentage correct prediction for shape-based technique is about $82.1 \%$.

\section{References}

[1] E. Oberg, F. Jones, H. Horton and H. Ryffel, 27th Edition Machinery's Handbook, New York: Industrial Press Inc, 2004.

[2] M. Kato, H. Takahashi and H. Hoshino, "Screw Inspection Device". United States Patent 4,457,622, 3 July 1984

[3] D. Thompson and W. Redmond, "Automated Fastener Inspection
System". United States of America Patent 4,823,396, 1989.

[4] M. Ekdahl, S. Oreg., J. T. H. Bellevue and Kirk.United States of America Patent 5727300, 1998.

[5] Teuku Muhammad Johan and Anton Satria Prabuwono, "Recognition of Bolt and Nut using Artificial Neural Network," in 2011 International Conference on Pattern Analysis and Intelligent Robotics, Putrajaya, 2011.

[6] N. Sephus, S. Bhagavatula, P. Shastri and E. Gabriel, "An IndustrialStrength Pipeline For Recognizing Fasteners," in 2015 IEEE 14th International Conference on Machine Learning and Applications, Atlanta, 2015.

[7] "MathWorks," The MathWorks , Inc., 2016. [Online]. Available: http://www.mathworks.com/help/images/ref/regionprops.html. [Accessed 23 June 2016].

[8] Mokhairi Makhtar, Nur Shazwani Kamarudin, Syed Abdullah Fadzli and Mohd Fadzil Abdul Kadir, "The Contribution of Feature Selection and Morphological Operation For On-Line Business System's Image Classification," International Journal of Multimedia and Ubiquitous Engineering, vol. 10, no. 11, pp. 303-314, 2015.

[9] S. Mahajan, A. Das and H. K. Sardaka, "Image acquisition techniques for assestment of legume quality," Trends in Food Science \& Technology, no. 42, pp. 116-133, 2015.

[10] S. Rathore, M. Hussain and A. Khan, "Automated colon cancer detection using hybrid of novel geometric features and some traditional features," Computers in Biology and Medicine, no. 65, p. 279-296, 2015.

[11] B. Shrestha, Classification of Plants Using Images of Their Leaves, North Carolina: Appalachian State University, 2010.

[12] Y. Mingqiang, K. Kpalma and J. Ronsin, "A Survey of Shape Feature Extraction Techniques," in Pattern Recognition Techniques, Technology and Applications, Rennes, InTech, 2008, p. 51.

[13] A. Khotanzad and Y. Huahong, "Rotation Invariant Pattern Recognition Using Zernike Moments," Pattern Recognition, vol. 9, pp. 326-328, 1988.

[14] W. Weizhuo , J. Mottershead and M. Cristinel, "Mode-shape recognition and finite element model updating using the Zernike moment descriptor," Mechanical Systems and Signal Processing, vol. 23, no. 7, pp. 2088-2112, 2009.

[15] A. Broumandnia and J. Shanbehzadeh, "Fast Zernike wavelet moments for Farsi character recognition," Image and Vision Computing, vol. 25, no. 5, pp. 717-726, 2007.

[16] A. Tahmasbi, F. Saki and S. Shokouhi, "Classification of benign and malignant masses based on Zernike moments," Computers in Biology and Medicine, vol. 41, no. 8, pp. 726-735, 2011.

[17] P. Wellner, "Adaptive Thresholding for the," Xerox, Cambridge, 1993.

[18] B. Karlik and A. V. Olgac, "Performance Analysis of Various Activation Functions in," International Journal of Artificial Intelligence And Expert Systems (IJAE), vol. 1, no. 4, p. 113, 2011.

[19] Laerd, "Laerd Statistics," Lund Research Ltd, 2013. [Online]. Available: https://statistics.laerd.com/spss-tutorials/kruskal-wallis-htest-using-spss-statistics.php. [Accessed 3 July 2016]. 
Proc. of Sixth International Conference On Advances in Computing, Electronics and Electrical Technology - CEET

2016Copyright (C) Institute of Research Engineers and Doctors. All rights reserved. ISBN no. 978-1-63248-109-2 doi: 10.15224/ 978-1-63248-109-2-17

About Author (s):

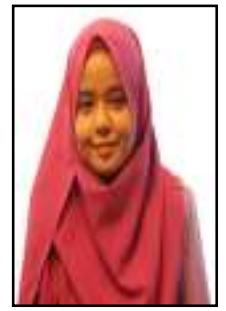

Nur Diyanah Mustaffa Kamal is an electrical engineering master degree student from Universiti Teknologi Mara. She obtained her bachelor's degree from Universiti Malaysia Perlis. Her main focus is on fastener recognition.

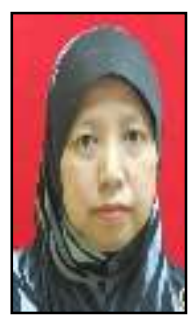

Dr Nor'aini Jalil is an associate professor at Universiti Teknologi Mara in electrical engineering faculty. Topics she mostly interested on are Zernike moments, orthogonal moments, and classification of iris region.

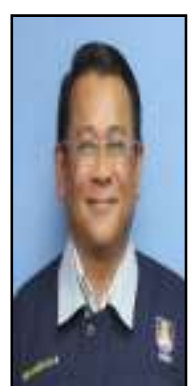

Dr Hadzli Hashim was a former associate professor at faculty of electrical engineering at Universiti Teknologi Mara. Topics he is mostly interested on are RGB color extraction of psoriasis lesion, medical skin disease imaging system and classification of rubber seed clones. 